\title{
Acknowledgement to Reviewers of Inorganics in 2019
}

Inorganics Editorial Office

MDPI, St. Alban-Anlage 66, 4052 Basel, Switzerland

Published: 17 January 2020

The editorial team greatly appreciates the reviewers who have dedicated their considerable time and expertise to the journal's rigorous editorial process over the past 12 months, regardless of whether the papers are finally published or not. In 2019, a total of 140 papers were published in the journal, with a median time to first decision of 14 days and a median time from submission to publication of 37 days. The editors would like to express their sincere gratitude to the following reviewers for their generous contribution in 2019:

\begin{tabular}{|c|c|}
\hline Accardo, Grazia & Bendix, Jesper \\
\hline Achelle, Sylvain & Bernstein, Elliot R. \\
\hline Adonin, Sergey A. & Berreau, Lisa M. \\
\hline Åkermark, Björn & Bilyachenko, Alexey N. \\
\hline Akitsu, Takashiro & Bini, Marcella \\
\hline Aksenov, Sergey & Bizzarri, Claudia \\
\hline Aldrich-Wright, Janice & Blower, Philip J. \\
\hline Altomare, Marco & Bochmann, Manfred \\
\hline Álvarez, Eleuterio & Boeré, René \\
\hline Amanda, Lumsden & Bogdanovskaya, Vera \\
\hline Andruh, Marius & Bogliotti, Nicolas \\
\hline Anthony, Ben & Bokach, Nadezhda \\
\hline Arion, Vladimir & Borbas, K. Eszter \\
\hline Arosio, Paolo & Boronin, Andrei I. \\
\hline Ashok Kumar, Meiyazhagan & Borowski, Tomasz \\
\hline Atabaev, Timur & Boschi, Alessandra \\
\hline Atuchin, Victor V. & Boston, Rebecca \\
\hline Avdeeva, Varvara & Bouropoulos, Nikolaos \\
\hline Aymard, Luc & Braband, Henrik \\
\hline Baker, Robert & Bremholm, Martin \\
\hline Ball, Zachary T. & Briois, Pascal \\
\hline Banach, Marcin & Broere, Daniel \\
\hline Bandiello, Enrico & Bruce, Alice \\
\hline Baranyai, Zsolt & Brugna, Myriam \\
\hline Baron, Marco & Burke, Benjamin \\
\hline Baschieri, Andrea & Burke, Richard \\
\hline Beckett, Michael A. & Bux, Sabah \\
\hline Belkova, Natalia V. & Buxton, Samuel \\
\hline Belokoneva, Elena & Caballero, Antonio \\
\hline
\end{tabular}


Cabot, Pere L.

Capacchione, Carmine

Cardinale, Jens

Casado, Miguel

Casini, Angela

Cavazza, Christine

Cepeda, Javier

Champagne, Benoît

Charnay, Clarence

Chen, Chien-Hong

Cheng, Yang-Tse

Chiang, Ming-Hsi

Chivers, Peter

Cho, Dae Won

Chu, Jiaxiang

Ciurli, Stefano

Clark, Tim

Cohen, Ronald

Conejero, Salvador

Correia, Ilidio

Correia, João D. G.

Costantino, Ferdinando

Croissant, Jonas G.

Crowley, James

Czlonkowska, Anna

Czujko, Tomasz

Darensbourg, Marcetta

Darling, Seth B.

De Munno, Giovanni

De Stefano, Luca

Della Ca', Nicola

Denat, Franck

Desmarets, Christophe

Di Bella, Santo

Diana, Rosita

Doert, Thomas

Domagała, Małgorzata

Dorta, Reto

Duan, Lian

Eaton, Gareth

Ekaterina, Bartashevich

Ellison, Paul

Faller, Peter
Fernández-Moreira, Vanesa

Ferrer, Isabel

Fierro, Vanessa

Filippov, Oleg A.

Findlater, Michael

Florea, Mihaela

Florindo, Pedro R.

Fornasiero, Paolo

Fox, Mark A.

Frontera, Antonio

Furia, Emilia

Gambini, Marco

García-Lastra, Juan María

Garroni, Sebastiano

Gas, Piotr

Geletii, Yurii

Gelman, Dmitri

Genninati Crich, Simonetta

Geraldes, Carlos

Ghadwal, Rajendra

Gimeno, Concepción

Giuseppe, Zampella

Glover, T. Grant

Gorte, Raymond J.

Grasso, Salvatore

Graton, Jérôme

Grisi, Fabia

Gulino, Antonino

Gunner, Marilyn R.

Gurzhiy, Vladislav

Haber, Joel A.

Hadlington, Terrance

Hallmann, Lubica

Han, Heyou

Hausinger, Robert

Hawes, Chris S.

Haynes, Anthony

Heck, Jürgen

Hermann, Andreas

Hermann, Petr

Hirahara, Masanari

Hirasawa, Noriyasu

Hix, Gary 
Hofer, Tim

Hogarth, Graeme

Honek, John

Horch, Marius

Horchidan, Nadejda

Horeglad, Paweł

Hosmane, Narayan

Hoster, Harry E.

$\mathrm{Hsu}, \mathrm{Fu}-\mathrm{Yin}$

Hsu, Yung-Jung

Hureau, Christelle

Hutchins, Kristin M.

Idogawa, Masashi

Inaba, Satoshi

Infantes-Molina, Antonia

Isobe, Hiroshi

Ito, Jun-ichi

Janczarek, Marcin

Jeong, Jae Min

Jones, Christopher E.

Junk, Peter

Kageyama, Hiroshi

Kameo, Hajime

Karcz, Dariusz

Kareiva, Aivaras

Kays, Deborah

Kaznelson, Boris

Keith, Jason M.

Kennedy, Brendan

Kerridge, Andrew

Khan, Mohammad Ehtisham

Kharisov, Boris I.

King, Paul

Klein, Axel

Klug, Dennis D.

Knölker, Hans-Joachim

Ko, Jiunn- Liang

Kokado, Kenta

Konarova, Muxina

Kostin, Gennadiy A.

Kotek, Jan

Kovács, Zoltán

Kraemer, Tobias
Kraka, Elfi

Kralj, Slavko

Kritchenkov, Andreii S.

Krylov, Alexander S.

Kukułka, Wojciech

Kumagai, Tomohisa

Lacerda, Sara

Lacroix, Pascal

Lauterbach, Lars

Lebeau, Bénédicte

Lee, Bang Yeon

Lee, Chia-Hung

Lee, Jong-Dae

Leimkuehler, Silke

Leong, David T.

Liddle, Steve

Lin, Kuo-Shyan

Linert, Wolfgang

Liu, Shuang

Lubomirsky, Igor

Luliński, Sergiusz

Lutsenko, Svetlana

Madsen, Robert

Mahmudov, Kamran T.

Małecka, Magdalena

Manayil, Jinesh C

Manzhos, Sergei

Marchetti, Fabio

Marek, Tkacz

Marín, Josefa Isasi

Martín, José I

Martínez-Lillo, José

Martins, Andre F.

Martins, Luísa Margarida

Martin-Vaca, Blanca

Massoud, Salah

Matikonda, Siddharth

Matilla-Hernández, Antonio

Mazzeo, Mina

McDonagh, Birgitte Hjelmeland

McMorran, David

Meier-Menches, Samuel M.

Meyer, Franck 
Michalke, Bernhard

Michalska-Domańska, Marta

Mills, David

Min, Byeong June

Miroslaw, Barbara

Miyazaki, Toshiki

Mondal, Kunal

Mondal, Mukulesh

Morrow, Janet R.

Mrówczyński, Radosław

Murray, Keith

Musiani, Francesco

Muzykantov, Vladimir R.

Nguyen, Minh Tho

Niewa, Rainer

Nikolaevskii, Stanislav A.

Nilges, Tom

Nilsen, Ola

Ocampo-García, Blanca Elí

Ogawa, Akiya

Oh, Jae-Min

Ohmura, Satoshi D.

Opazo, Carlos

Oreshonkov, Aleksandr

Orue, Inaki

Oswald, Iain

Owen, Gareth

Palella, Alessandra

Palinko, Istvan

Panich, Alexander M.

Pantazis, Dimitrios A.

Papa, Elettra

Papagiannopoulou, Dionysia

Papavasiliou, Joan

Parac-Vogt, Tatjana N.

Parfenova, Lyudmila

Parra, Ruben

Paschoal, C.W.A.

Paulo, António

Pedersen, Henrik

Pérez-Moreno, Javier

Perlepes, Spyros P.

Pla-Quintana, Anna
Poddel'sky, Andrey

Polisadova, Elena

Prabhakaran, Dharmalingam

Pushkar, Yulia

Puttreddy, Rakesh

Que, Emily L.

Remhof, Arndt

Riccò, Raffaele

Rigamonti, Luca

Roemelt, Michael

Roger, Julien

Ruiz, Miguel A.

Saielli, Giacomo

Sánchez, Goar

Santoni, Marie-Pierre

Sathekge, Mike

Sato, Toyoto

Schöllhorn, Bernd

Schwalbe, Matthias

Sheehan, Stafford W.

Shenderovich, Ilya G.

Shevelkov, Andrei V.

Sivaev, Igor B.

Sontakke, Atul D.

Sóvágó, Imre

Spingler, Bernhard

Stamatatos, Theocharis C.

Stone, Kari

Stripp, Sven T.

Sukhikh, Taisiya

T. Mague, Joel

Tabernero, Vanessa

Taddei, Marco

Takao, Toshiro

Takaya, Jun

Takeda, Youhei

Takeguchi, Tatsuya

Tang, Cheng-Ming

Tard, Cédric

Tavakkoli, Mohammad

Trujillo, Cristina

Tsai, Hui-Lien

Tubaro, Cristina 
Ueda, Tadaharu

Van Dam, R. Michael

Van Der Vlugt, Jarl Ivar

Varadwaj, Pradeep R.

Vedernikov, Andrei N.

Vinyard, David

Virgili, Tersilla

Vogiatzis, Konstantinos

Wagler, Jörg

Walton, Richard

Wang, Di-Yan

Wang, Jian

Wang, Junjie

Wann, Derek

Waterman, Rory

Weigand, Wolfgang

Wieser, Michael E.

Wikström, Mårten

Wilson, Justin J.
Worrall, Jonathan A. R.

$\mathrm{Wu}$, Ming-Chung

$\mathrm{Wu}$, Yuchuan

Xia, Tian

Xiong, Hao

$\mathrm{Xu}$, Chun

$\mathrm{Xu}$, Jian

$\mathrm{Xu}, \mathrm{Nan}$

Yakhvarov, Dmitry

Yamamoto, Yasunori

Yang, Chen-I

Yeo, Boon Siang

Yukl, Erik T.

Zeller, Matthias

Zhang, Xu

Zhou, Kun

Zima, Vitezslav

Zuev, Andrey

(C) 2020 by the authors. Submitted for possible open access publication under the terms and conditions of the Creative Commons Attribution (CC BY) license (http://creativecommons.org/licenses/by/4.0/). 